\title{
Production of Blood Serological Test Device
}

National Cancer Institute

\section{Source}

National Cancer Institute. Production of Blood Serological Test Device. NCI Thesaurus. Code C112942.

A procedure to manufacture devices or components used in blood serological testing. 